\title{
TRICUSPID INCOMPETENCE
}

\author{
BY \\ OTTAR MÜLLER* AND JOHN SHILLINGFORD † \\ From the Department of Medicine, Postgraduate Medical School, Hammersmith, London
}

Received January 1, 1954

Tricuspid incompetence seems worthy of further analysis by means of pressure recordings from the right heart related to phlebography and phonocardiography, in view of the varied opinions expressed regarding its frequency and the diagnostic criteria. As the study proceeded it was clear that our diagnostic criteria were changing and that little purpose would be served by a review of past records; for this reason, only cases studied during the last year are being reported and the number (21) is not yet adequate for a full review of all aspects of the condition. It is felt that the findings may have some bearing on the topical general problem of incompetence of the atrioventricular valves.

\section{Historical Note}

In $1835 \mathrm{King}$ placed in the museum of Guy's Hospital more than 300 specimens to illustrate what he assumed was the safety valve function of a tricuspid regurgitation from the right ventricle and stated in 1837: "The veins being more or less influenced by their own number and capacity, by the position of the body, by cold, compression, repletion and respiration, the blood is brought to, or collected in, the right ventricle in varying quantities, and on the occasions of the most copious influx the cavities become distended; upon which the curtains of the tricuspid valve are drawn aside, an aperture of reflux is produced and the force of the ventricle is diverted from the pulmonary circulation at the moment when the lungs might otherwise be overwhelmed." The same author differentiated between the arterial and venous pulses in the neck and gave an extensive description of the anatomy of the tricuspid valve. Benson (1836) noted that the systolic venous pulsation in the neck occurred slightly later in time than the arterial pulse in a case which at necropsy showed organic tricuspid incompetence. Adams (1827), Davies (1827), Ward (1832), and Graves (1833) all reported cases in which they associated systolic venous pulsation in the neck with tricuspid incompetence.

Experimental tricuspid incompetence in dogs was produced by Rosenbach (1878) who recorded that avulsion of the tricuspid valve was followed by a loud systolic murmur, but was unable to find any relationship between the extent of the injury and the loudness of the murmur. Further experiments in dogs were made by Rihl (1907) who found that the production of tricuspid incompetence caused a ventricular type of venous pulse when the lesions were extensive; small lesions, however, did not produce this type of pulse.

Mackenzie (1908), on clinical grounds and on the evidence of the polygraph tracings of the jugular venous pulse, considered tricuspid incompetence to be a common condition and wrote:

"Although actual disease of the valve is rare, incompetence of the tricuspid orifice is extremely common, so common indeed that I am inclined to look upon the valves as being barely able to close the orifice properly."

* In receipt of a Norwegian State Grant.

$\dagger$ In receipt of a Grant from the Medical Research Council. 
Laubry and Routier (1928) and, more recently, Soulié et al. (1952) have taken a more conservative view of the frequency and have pointed out that the tricuspid systolic murmur is often difficult to distinguish from those murmurs produced elsewhere in the heart and that, as Mackenzie was aware, pulsations of the neck veins in auricular fibrillation may be similar to those found in tricuspid incompetence.

\section{Methods}

Twenty-one patients who, after a full clinical study, were judged to show tricuspid incompetence are reported. Phonocardiograms were taken by means of a crystal microphone connected either to a Cambridge galvanometer or to a balanced amplifier and Elmquist triplex electrocardiographic recorder. The frequency response was logarithmic up to 800 cycles a second. Simultaneous electrocardiograms were taken in all.

The jugular venous pulse was recorded in all patients by means of a small suction cup applied to the neck over the internal jugular vein, and connected by a short rubber tube to either a piezo-electric microphone or a Frank segment capsule, the moving light beam from which illuminated a variable area on a photoelectric cell. Impulses from this were amplified by a linear amplifier with variable electronic damping and recorded on the Elmquist electrocardiograph.

Intracardiac pressure recordings were made in patients where cardiac catheterization was performed either for diagnostic purposes or prior to operation for mitral stenosis. A Statham strain gauge was used, amplified by a direct current amplifier connected to the Elmquist electrocardiograph. In the earlier cases a 0-4-1b. gauge was used but this was found to be overdamped for accurate atrial tracings with a catheter size under No. 8 and in the later patients a 0-15-1b. gauge with increased electronic amplification was substituted.

\section{General Clinical Data on the Patients Studied}

Table I shows the types of cardiac disease in which the lesion was found. Dyspnœa on exertion, to a lesser or greater degree was present in all our patients. In many of them a striking feature was the slight degree of dyspnœa when compared with the greatly raised venous pressure. In one mitral stenotic the development of gross tricuspid incompetence led to a significant improvement of effort tolerance. Peripheral œdema was severe in six, moderate in eight, slight in three, and absent in four patients. Pulsation of the usually enlarged liver closely paralleled the amplitude of pulsation in the neck veins and was noted in sixteen; in none of these was there much jaundice but an icteric tinge was present in four. Sherlock (1951) noted that 5 out of 16 patients with cardiac jaundice (serum bilirubin more than $2 \mathrm{mg}$. $/ 100 \mathrm{ml}$.) had tricuspid incompetence.

TABLE I

CAUSAL Conditions OF TRICUSPID INCOMPETENCE

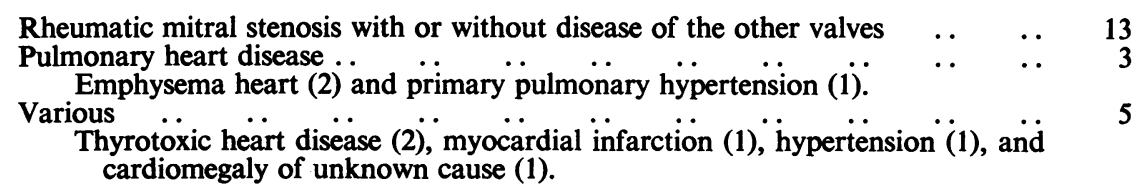

The venous pressure was invariably raised, being over $8 \mathrm{~cm}$. above the sternal angle in eleven, from 4 to $8 \mathrm{~cm}$. in six, and 2 to $4 \mathrm{~cm}$. in four patients. Auricular fibrillation was present in seventeen and sinus rhythm in four. One patient with chronic rheumatic heart disease and mitral stenosis in whom tricuspid incompetence could not be demonstrated when in sinus rhythm developed gross tricuspid incompetence on a change of rhythm to auricular fibrillation; subsequent reversion to sinus rhythm was accompanied by the disappearance of tricuspid incompetence.

\section{THE MURMURS}

To overcome difficulties in the interpretation of murmurs we have separated two groups, those with no other demonstrable valve lesions and those with associated valve lesions and complicating murmurs. The first group consists of seven cases of pulmonary, hypertensive, and thyrotoxic 
heart disease. A systolic murmur was heard in six, loud and widely-distributed in one, soft and medium pitched in five; it was absent in one. The distribution of the murmur in these patients is shown in Fig. 1 where it will be seen that it is best heard to the left of, and adjacent to, the sternum
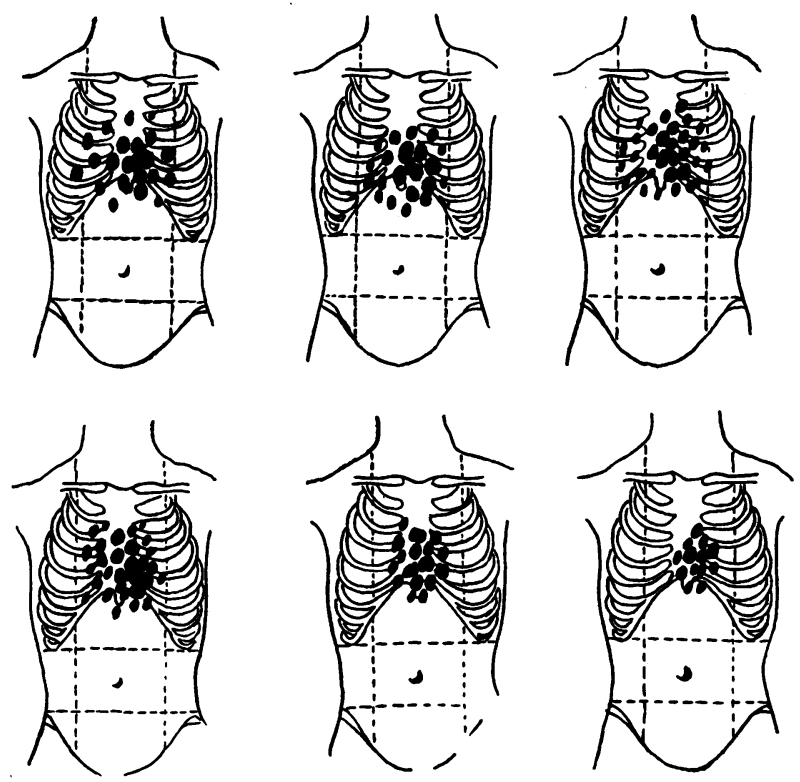

FIG. 1.-The distribution of the systolic murmur in six patients with tricuspid incompetence without other complicating murmurs.

in the fourth interspace; radiation of the murmur was noted only a short distance towards the apex but was often marked to the right of, and below, the xiphisternum. In five patients the murmur was pansystolic in nature with an emphasis in early systole (Fig. 2 and 12), but in one there was an increase in the intensity towards the end of systole. In four where the patient was exercised the strength of the murmur was increased and fell to its original level of intensity a few minutes after the exertion was stopped (Fig. 3). An increase in the intensity of the murmurs in deep inspiration, as was described by Carvallo (1946), was noted in all cases where other valve lesions were absent (Fig. 4) and this was associated with an increase in the amount of tricuspid regurgitation as shown

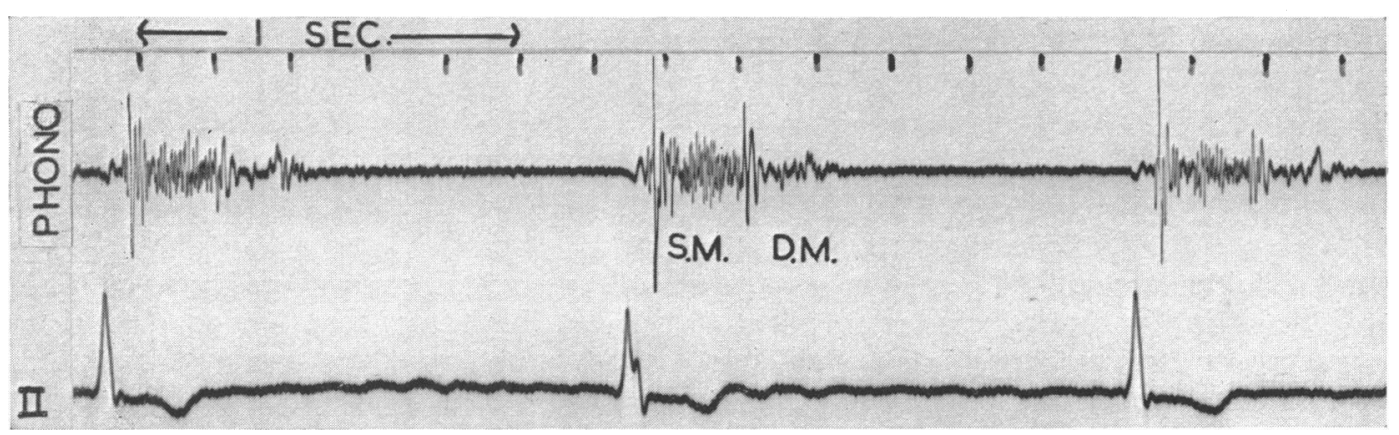

FIG. 2.-The systolic and early diastolic murmurs of tricuspid incompetence. Medium frequency record $\mathbf{O}$ taken at the lower end, and slightly to the left of, the sternum. 


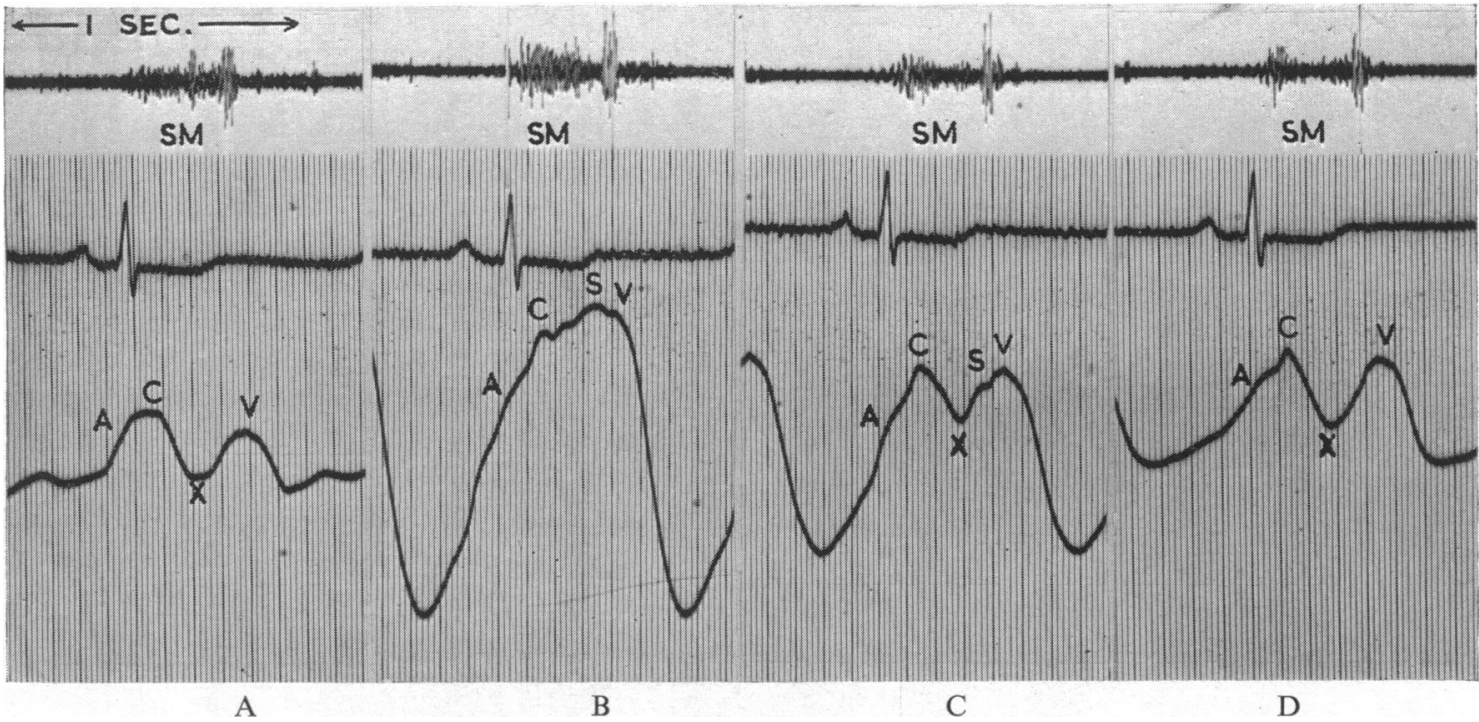

FIG. 3.-The effect of exercise on the systolic murmur and the jugular venous pulse in a patient with tricuspid incompetence. (A) Before exercise, (B) after one minute of exercise, (C) two minutes after cessation of exercise, and (D) five minutes after exercise. Exercise causes the development of the systolic wave, $\mathrm{S}$, and an increase in the systolic murmur.

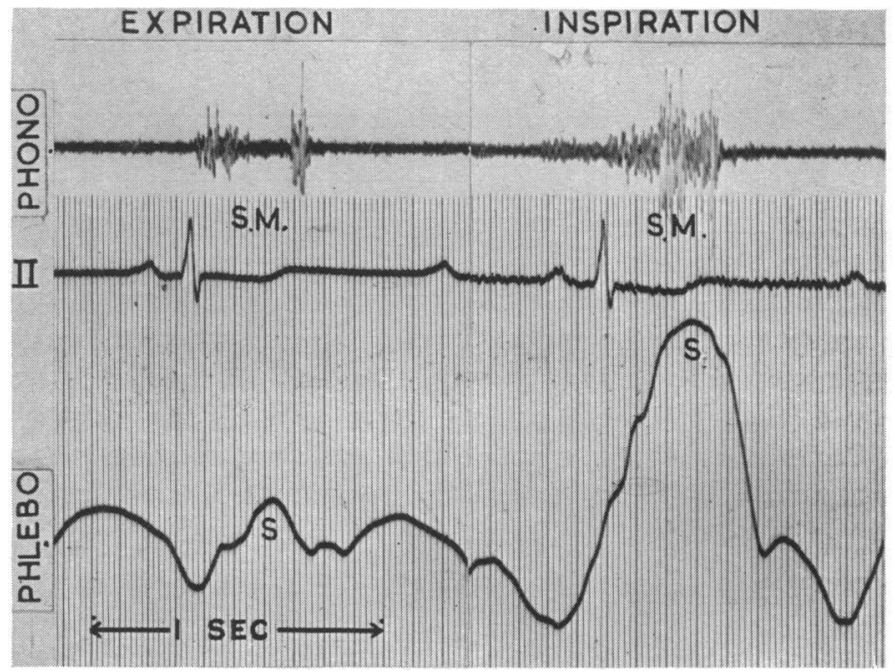

Fig. 4.-The effect of respiration on the murmur and the jugular phlebogram in a patient with tricuspid incompetence. Inspiration increases the positive systolic wave, $S$, and the intensity of the systolic murmur.

by the simultaneous phlebogram. In some cases of tricuspid incompetence in severe chronic rheumatic heart disease with gross enlargement of the heart, inspiratory accentuation of the murmur was not present, and in these it was not possible to alter the pattern of the jugular venous pulse on deep breathing. In two cases the systolic murmur was transient in nature and rapidly disappeared following clinical improvement. In cases with auricular fibrillation the murmur was often increased in the beats following the long pause, possibly because of greater regurgitation from increased end diastolic filling (Fig. 5). 


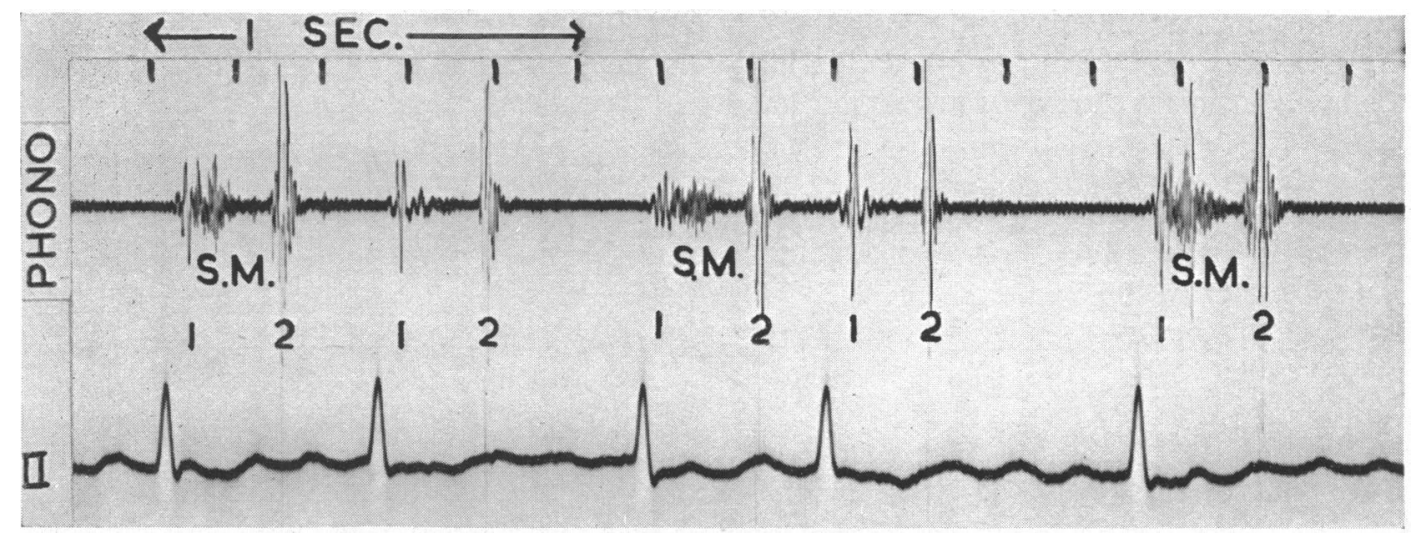

FIG. 5.-The systolic murmur of tricuspid incompetence in the presence of auricular fibrillation. There is an increase in the intensity of the murmur after the long diastolic pauses.

In the second group of 13 patients other valve lesions were present. It was possible to hear a distinctive murmur in eight, in three of whom it was accompanied by a thrill; in the other five the murmurs in the tricuspid and apical areas could not be distinguished with certainty one from another.

Messer et al. (1950) demonstrated the presence of a short low pitched diastolic murmur, occurring immediately after the second sound in patients with tricuspid incompetence. We have heard and recorded this murmur in nine patients (Fig. 2): all these nine had other signs of a severe tricuspid leak. This murmur was well heard and recorded in one where at subsequent necropsy no disease of the valve was present, beyond gross dilatation of the valve ring.

\section{The Phlebogram}

Jugular phlebograms were made from all our patients. The normal jugular pulse shows a positive wave ( $a$ wave) beginning just before, and reaching its peak, with atrial systole; the first part of the wave is due to passive filling of the right atrium and later to atrial contraction. Following this the negative, or $x$ wave, occurs which is thought to be due to atrial diastole and the descent of the base of the heart. In many tracings the underlying carotid artery produces an interference wave in this part of the curve. Towards the end of systole a new positive wave ( $v$ wave) appears due to the passive filling of the atrium, continuing until the opening of the tricuspid valve. The pattern in auricular fibrillation in the absence of tricuspid incompetence is essentially the same except that atrial contraction is no longer represented on the summit of the $a$ (filling) wave. The general pattern of the jugular phlebogram follows the atrial pressure curves very closely when the jugular veins are well filled during the whole of the cardiac cycle; allowance must be made, however, for the differences in methods of recording and for a slight time delay of the pressure wave between the atrium and jugular vein. In the grosser examples of tricuspid incompetence (16) the normal systolic dip was obliterated or reversed by the positive systolic wave (Fig. 6). In less severe cases there was an incomplete obliteration, in varying degrees, of the systolic dip. On the stroke of the wave following the $x$ descent a small discrete wave was often seen to rise to the level of, or higher than the normal $v$ wave. This effect could be well demonstrated in patients where an increase in the tricuspid leak was produced by exercise. We believe that this is the earliest phlebographic sign of tricuspid incompetence, and that it is not possible to distinguish such a change by simple observation of the veins of the neck.

The effect of exercise on the amount of tricuspid regurgitation was well marked in many of our patients. Two minutes' exercise by moving the legs against resistance while lying on the couch produced an increase in the positive systolic wave which correlated with an increase in the systolic murmur in the tricuspid area. During the period of rest following the exercise this wave was seen to 
decrease, as did the intensity of the murmur (Fig. 3). In cases of auricular fibrillation an increase in the positive systolic wave was noted after long diastolic pauses and presumably this resulted from the same factors that caused an increased intensity in the murmur (Fig. 7).

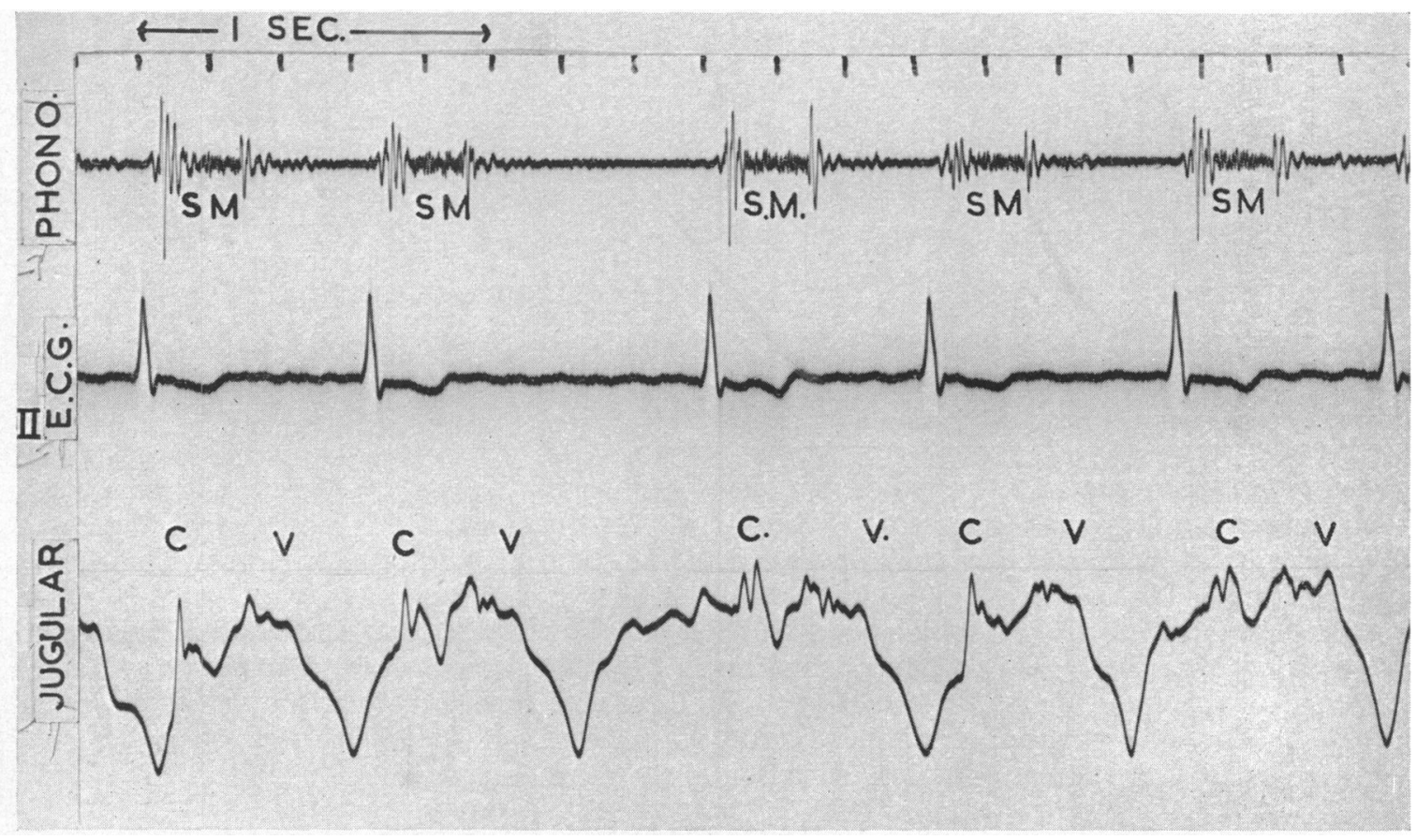

FIG. 6.-The jugular phlebogram in a patient with tricuspid incompetence showing the positive systolic wave and coarse vibrations between $c$ and $v$.

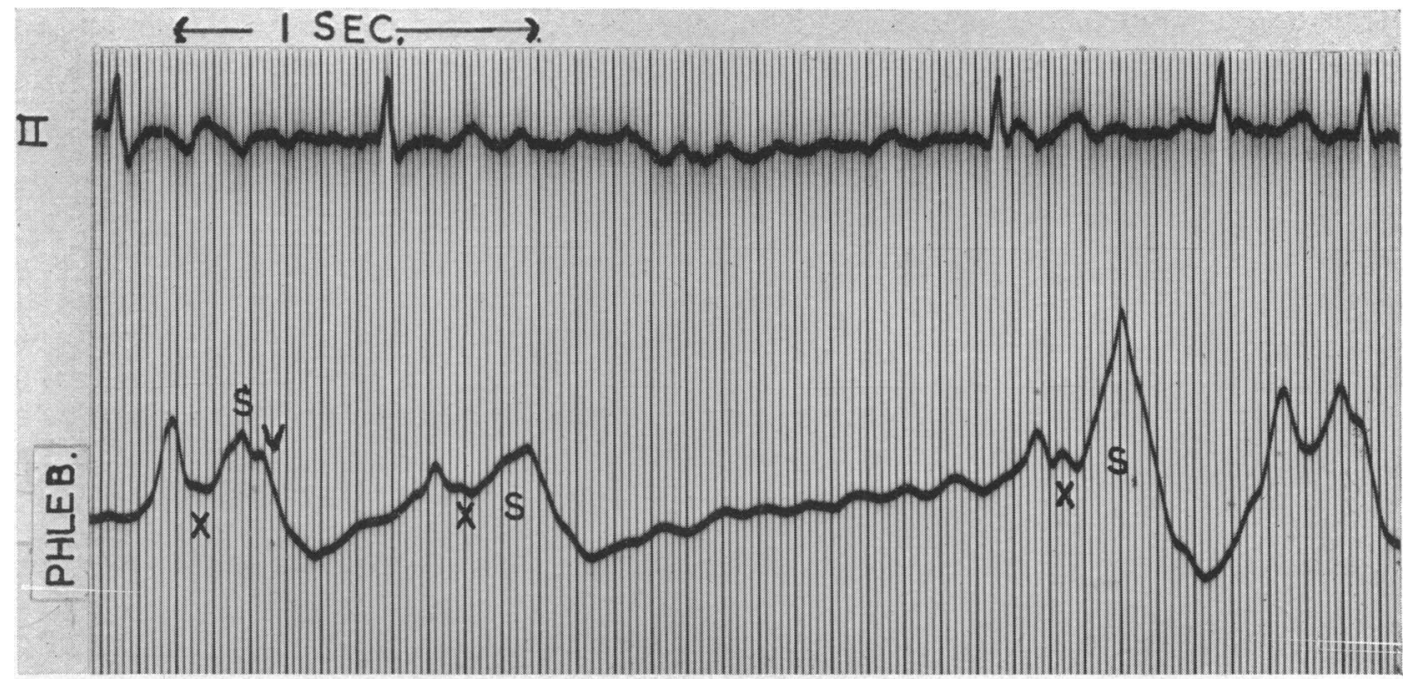

FIG. 7.-The jugular phlebogram in a case of tricuspid incompetence in the presence of auricular fibrillation, showing an increase in the systolic wave, $\mathbf{S}$, after a long pause. 
The Right Atrial Pressure Records

Cardiac catheterization was performed in 12 of our patients. In five, pulmonary arterial and right ventricular pressure tracings were made as well as those from the right atrium and superior vena cava. In one, differential pressure recordings between the right atrium and superior vena cava were made. Table II summarizes the levels of intracardiac pressure and cardiac output obtained by the direct Fick method. In all cases there was a raised right atrial pressure, the mean varying from 9 to $25 \mathrm{~mm}$. of mercury. Atrial systolic pressures varied from 12 to $43 \mathrm{~mm}$. and the end diastolic from 8 to $22 \mathrm{~mm}$. The ratio of the right atrial and right ventricular systolic pressures varied between $1 / 1.3$ and $\mathrm{i} / 4$ and we believe that this ratio falls with increasing tricuspid incompetence. Pressures were measured from $5 \mathrm{~cm}$ below the sternal angle.

TABLE II

Physiological Findings in Cases of Tricuspid Incompetence

\begin{tabular}{|c|c|c|c|c|c|c|}
\hline \multirow{2}{*}{ Case } & \multicolumn{3}{|c|}{ R.A. pressure (mm. $\mathrm{Hg})$} & \multicolumn{2}{|c|}{ R.V. pressure (mm. Hg) } & \multirow{2}{*}{$\begin{array}{l}\text { Cardiac output } \\
\text { litres/min. }\end{array}$} \\
\hline & Systolic & Diastolic & Mean & Systolic & Diastolic & \\
\hline $\begin{array}{l}\text { M.P. } \\
\text { D.B. } \\
\text { G.S. } \\
\text { L.F. } \\
\text { A.M. } \\
\text { M.R. } \\
\text { F.M. } \\
\text { E.W. } \\
\text { W.L. } \\
\text { J.B. }\end{array}$ & $\begin{array}{l}43 \\
40 \\
30 \\
28 \\
25 \\
24 \\
12 \\
14 \\
12 \\
18\end{array}$ & $\begin{array}{c}22 \\
16 \\
12 \\
13 \\
14 \\
12 \\
4-8 \\
5-12 \\
5-10 \\
0-8\end{array}$ & $\begin{array}{l}25 \\
20 \\
20 \\
20 \\
18 \\
17 \\
10 \\
11 \\
9 \\
14\end{array}$ & $\begin{array}{l}52 \\
\overline{-} \\
\overline{86} \\
\frac{45}{45} \\
75\end{array}$ & $\begin{array}{l}\frac{22}{二} \\
\overline{18} \\
\frac{22}{18}\end{array}$ & $\begin{array}{l}5.5 \\
3.5 \\
2.0 \\
3.2 \\
2.1 \\
5.5 \\
3.2 \\
6.4 \\
3.0 \\
2.3\end{array}$ \\
\hline
\end{tabular}

The cardiac output varied between 2 and 6.4 litres a minute and did not appear to be related, in any way, to the degree of tricuspid regurgitation.

The atrial pressure curves can be divided into two groups. In the first ( 4 cases) the characteristic feature was a single positive systolic wave, sustained through ventricular systole, such that the atrial tracing resembled that obtained from the right ventricle (Fig 8B and 9B); on the upstroke

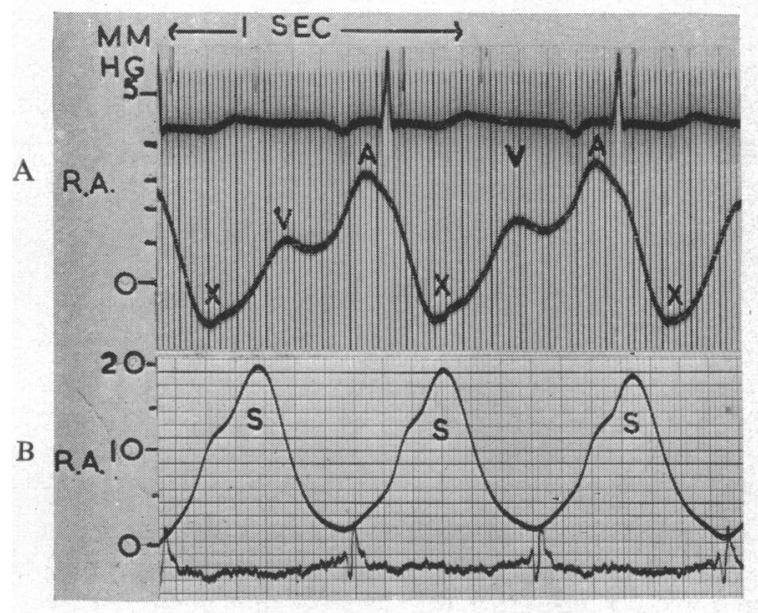

FIG. 8.-Right atrial pressure curves. (A) In a normal heart and (B) in a case of severe tricuspid incompetence; both in regular rhythm. The $\mathrm{X}$ descent has been replaced by a positive systolic wave, $S$.

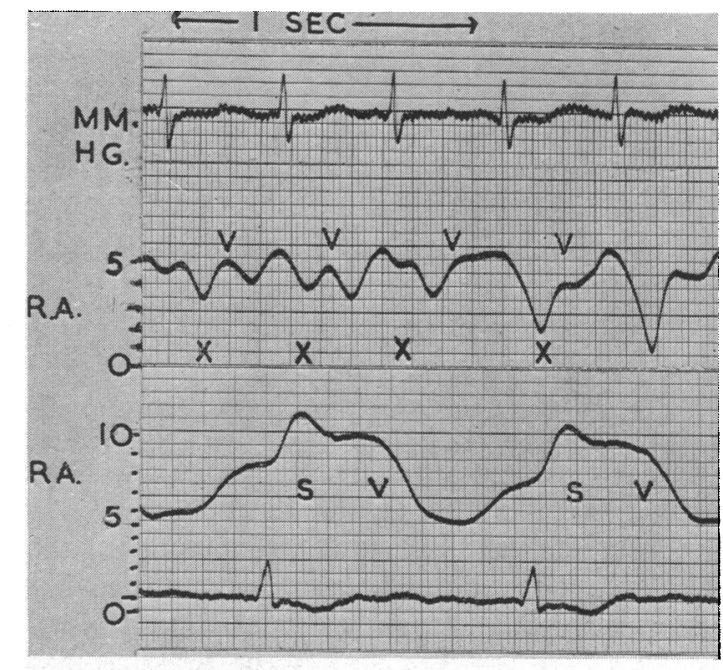

Fig. 9.-Right atrial pressure curves. (A) In a heart without tricuspid incompetence and (B) in one with tricuspid incompetence. Both cases are in auricular fibrillation. 
of this wave a small notch was found which was almost certainly due to the partial closing of the tricuspid valve. In the second group ( 8 cases) the normal systolic dip was altered by the presence of a positive deflection which in some cases could be distinguished from the $c$ and $v$ waves, and in others was seen to be merging into the $v$ wave and giving a plateau appearance or slightly convex curve. The latter effect was especially noticeable in tracings in which the damping was greatest. This wave began to rise, in some cases at the position of the $c$ wave, and in others it was delayed up to $0.1 \mathrm{sec}$. after it, with the production of varying degrees of $x$ descent. In the less damped curves coarse vibrations were seen during the systolic period (Fig. 10).

The height of the systolic wave was affected by the phase of respiration. Inspiration produced an increase in the positive deflection, in some cases producing a remarkable change from a systolic dip to a ventricular type of atrial pulse (Fig. 11). At the same time the peak of the systolic wave

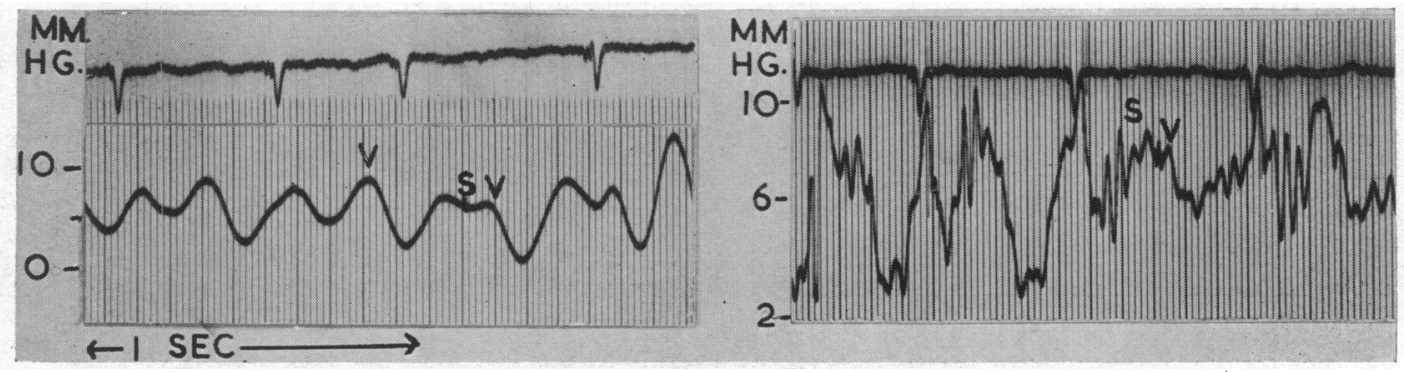

Fig. 10.-Right atrial pressure curves in a case of tricuspid regurgitation. (A) Damped and (B) undamped curves showing the positive systolic wave to be made up of coarse vibrations.

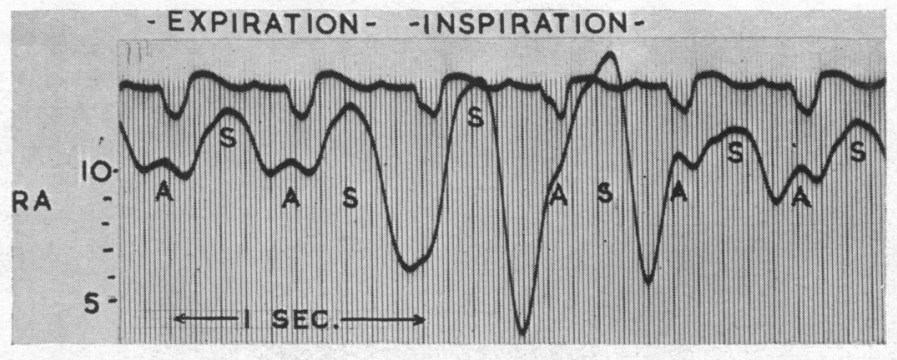

FIG. 11.-The effect of respiration on the right atrial pressure curves in tricuspid incompetence, showing a great increase in the positive systolic wave, $S$, in inspiration.

moved nearer to the first heart sound. Exercise also increased the degree of tricuspid regurgitation as shown by a decrease in the systolic dip when present, or by an increase in the positive systolic wave.

In auricular fibrillation the " descent of the base " $x$ dip is not so apparent as in normal rhythm because of the absence of atrial systole and diastole. However, the descent can clearly be seen in some cases of auricular fibrillation where the right atrial pressure is not greatly raised. In most instances of auricular fibrillation, where right atrial pressure is high some degree of tricuspid leak occurs, the leak increasing after the longest diastolic intervals. This is shown not only by the atrial pressure tracings and the jugular phlebograms but also by the variations in intensity of the systolic murmur heard in the tricuspid area. 
Differential pressure records between the right atrium and superior vena cava were made in one case to determine the direction of flow between the atrium and great veins during the cardiac cycle. The curve demonstrated that there was a reversed flow from the right atrium to the superior vena cava throughout systole and that this was greatest early in systole (Fig. 12). The greatest inflow from the superior vena cava to the right atrium occurred immediately after the end of systole; this variation in flow corresponded to the position of the systolic and early diastolic murmurs in the cardiac cycle and to the accentuation of the systolic murmur at the beginning of systole.

The pressure tracings taken from the superior vena cava resembled those taken from the right atrium. In gross cases of tricuspid incompetence the positive systolic wave was suddenly cut off on withdrawal of the catheter to the axillary vein and the pressure curves resembled those obtained in minor degrees of tricuspid leak (Fig. 13). This effect was thought to be due to withdrawal of the catheter past a relatively competent venous valve in the axillary vein.

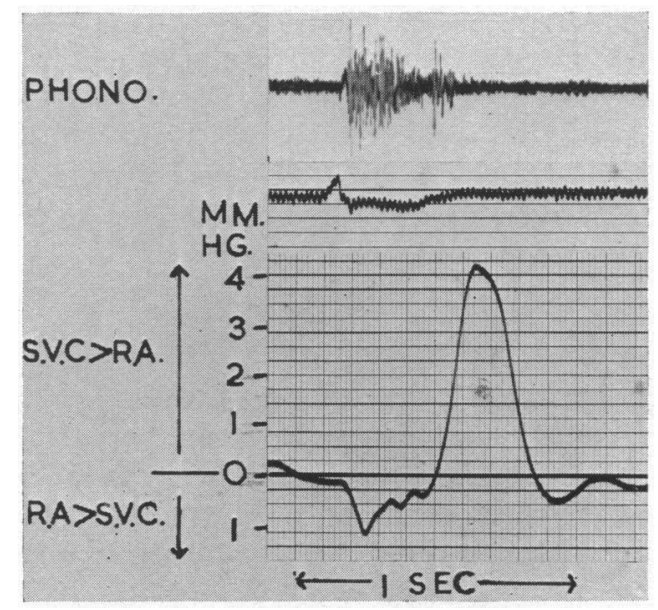

FIG. 12.-The differential pressure curve between the right atrium and superior vena cava. The part of the curve below the zero line represents an inverted pressure gradient. Note the relationship of the systolic murmur to this curve. The phonocardiogram was taken separately and superimposed.

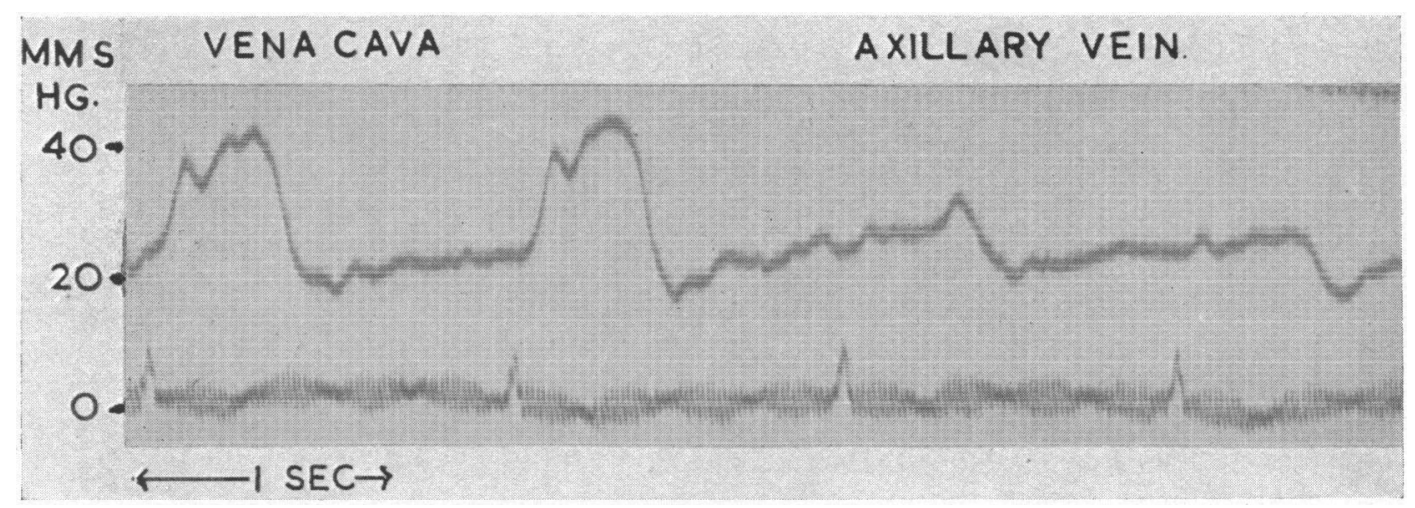

FIG. 13.-The effect of withdrawing the catheter past a venous valve in a case of tricuspid incompetence. The electrocardiogram is disturbed by electrical interference. 


\section{ELECTROCARDIOGRAPHY}

Four patients were in sinus rhythm and seventeen in auricular fibrillation. The electrocardiogram often suggested right ventricular preponderance-great in three cases, moderate in three, and slight in one. Right bundle branch block was present in four patients and in these it was complete in one and partial in three. No preponderance was present in seven, while two showed moderate left ventricular hypertrophy and one left bundle branch block.

\section{RADIOLOGY}

While the study of the left atrial movements has been thought important in the diagnosis of mitral incompetence the radiological study in tricuspid incompetence has been singularly disappointing. Twenty cases were examined. All the hearts were abnormal in shape and size. The right atrial border was prominent in twelve in the anterior-posterior view and in three the prominence was great. Examination for the pulsation of the auricular border and the superior vena cava was generally disappointing although in three cases a marked systolic expansion could be seen. On the other hand no pulsation was present in a case of gross and long standing tricuspid incompetence. The other contours of the heart were consistent with the underlying cardiac lesion. Hilar congestion was present in twelve patients and was conspicuously absent in six in whom the tricuspid regurgitation was severe and long standing.

\section{NECROPSY FINDINGS}

Four patients came to necropsy. The first in life showed a very severe tricuspid regurgitation. Heart weight $700 \mathrm{~g}$. with great hypertrophy and dilatation of both ventricles and the right atrium; tricuspid ring measured $16 \mathrm{~cm}$. but the valve cusps showed no organic disease.

The second had a severe tricuspid regurgitation in life which had been observed for several years. Heart weight $446 \mathrm{~g}$. with great dilatation of the right atrium and ventricle; stenosis of mitral valve with slight aortic stenosis; tricuspid ring $17 \mathrm{~cm}$. in circumference with no rheumatic involvement of the valve cusps.

The third had a moderately severe tricuspid regurgitation in chronic rheumatic heart disease. Heart weight $440 \mathrm{~g}$.; right ventricle and right atrium greatly hypertrophied and moderately dilated; stenosis of the mitral and aortic valves; tricuspid ring $12 \mathrm{~cm}$.; leaflets of tricuspid valve not thickened.

The fourth showed moderate tricuspid regurgitation in chronic rheumatic heart disease. Heart weight $560 \mathrm{~g}$.; right ventricle and right atrium greatly hypertrophied and dilated; tricuspid ring $15 \mathrm{~cm}$. and rheumatic involvement of tricuspid valve.

All these cases exhibited moderate or severe tricuspid incompetence in life as judged by the phlebographic and right atrial tracings and accompanied by a loud systolic murmur. These findings confirm our view that it is not possible to say whether there is involvement of the valve cusps by disease by clinical examination.

\section{Discussion}

Several authors, including King (1837) and Mackenzie (1908), have stressed the frequent occurrence of a tricuspid regurgitation in right ventricular failure. They have also emphasized the transient nature, in some cases, of such a leak. We have made similar observations and feel that on the evidence of the clinical findings and the phlebographic and right atrial pressure curves, some degree of tricuspid regurgitation is extremely common in all forms of cardiac failure.

The majority of our patients who showed tricuspid incompetence had rheumatic heart disease and this may be accounted for by the continuously high and often increasing load that develops on the right ventricle as contrasted with the episodic nature of pulmonary hypertension in pulmonary and hypertensive heart disease.

The systolic murmur, best heard at the lower end, and slightly to the left, of the sternum, appears to be one of the earliest clinical signs of tricuspid regurgitation although occasionally it may be 
absent with well marked tricuspid incompetence. In patients who have auricular fibrillation its intensity varies from beat to beat and may be absent in beats after short diastolic pauses although loud in other beats; this variation is probably due to different degrees of end diastolic filling of the right side of the heart. Mackenzie pointed out the transient nature of the murmur and our observations have confirmed not only the disappearance of the murmur on clinical improvement of the patient but its variation in intensity from day to day.

The systolic murmur may generally be differentiated from that of mitral incompetence by its distribution and by the effect of deep inspiration and expiration which has an opposite effect on the left side as compared with the right (Fig. 14). The murmur in tricuspid incompetence is often accentuated in early systole.

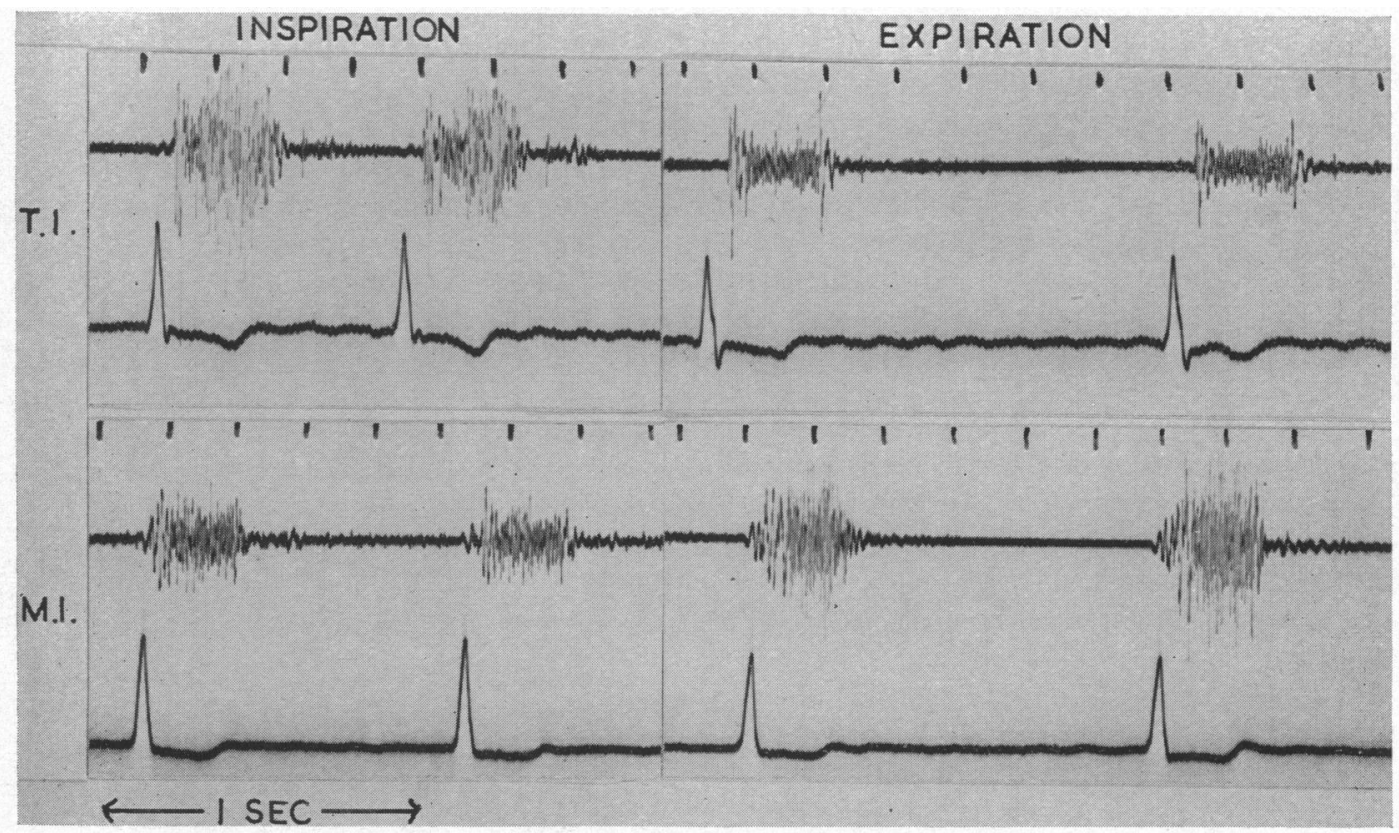

Fig. 14.-The effect of respiration on the murmurs of tricuspid and mitral incompetence.

The early, low pitched, diastolic murmur was an interesting finding in eight of our patients, and, as was shown by necropsy control, can occur in the absence of any organic disease of the valve cusps. It may be due to the large volume of blood passing from the tense right atrium at a high velocity through the tricuspid valve at the beginning of diastole.

A positive systolic wave in the internal jugular veins visible to the naked eye appears to be a later manifestation than the murmur; expansile pulsation of the liver, although not felt in all cases, was common.

The instrumental detection of a leak through the tricuspid valve is approached indirectly by studying (1) the effect of such a leak on the pressure changes in the right atrium, (2) the volume changes reflected in the jugular phlebogram, and (3) the directional changes in the blood flow in the right atrium and great veins by means of differential manometry.

Bloomfield et al. (1946) described the right atrial pressure curves in eight cases of tricuspid incompetence and showed the normal systolic dip to be replaced by a wave that had the form of a plateau or showed an upward convexity; the pressure level was higher than in the intervening presystolic interval and was sustained until the end of isometric relaxation. These authors considered that where there was heart failure without tricuspid incompetence the systolic dip was accentuated. 
Lagerlof and Werko (1948) made similar observations. McCord and Blount (1952) found in four patients, all with rheumatic heart disease and clinical evidence of tricuspid regurgitation, a positive wave in systole that could sometimes be increased by exercise: they thought that the amplitude of this wave was an indication of the severity of the lesion. Our findings agree with these and in addition we have found in most cases an increase in the regurgitation on deep inspiration.

Gerhardt (1902) described in detail various types of positive systolic waves seen in the phlebographic tracings from patients with tricuspid incompetence. He showed that this positive systolic wave could be maximal at any phase in systole and that sometimes small vibrations could be seen superimposed upon it. Rihl (1907) demonstrated a similar finding after experimental avulsion of the tricuspid valve in dogs. Later workers including Mackenzie (1908), Wiggers (1923), Teufl (1936), Groedel (1946), and Messer (1950) have also made similar observations. Our results confirm these and show that the phlebographic tracings from the internal jugular vein closely reflects the wave form of the right atrial pulse; the effect of inspiration and exercise was the same on both the jugular phlebograms and right atrial pulse curves.

Although functional tricuspid incompetence appears to be extremely common, organic lesions are relatively rare and we have not been able to distinguish with certainty the former from the latter by either clinical or instrumental observation. Some patients with tricuspid incompetence show remarkably little disability for long periods; this may be analogous to incompetence of other valves of the heart without actual cardiac failure. In the majority of patients, however, tricuspid incompetence develops as part of the progressive downhill course of cardiac disease.

\section{SUMMARY}

Twenty-one patients with tricuspid incompetence have been studied clinically and by phonocardiography, phlebographic recordings, and intracardiac catheterization.

The earliest clinical sign of tricuspid incompetence was the presence of a systolic murmur at the lower end of the sternum, increased by exercise and deep breathing, and of particular distribution and character. Other signs, in order of appearance, were systolic pulsation in the internal jugular veins and pulsation of the liver.

The character and timing of the murmur, as shown by the phonocardiogram, and the positive systolic wave in the jugular veins as recorded by the phlebogram have been described.

The intracardiac pressure curves, both single and differential, have been described and related to the clinical findings.

Radiology and electrocardiography have not contributed greatly to the diagnosis of tricuspid incompetence.

We should like to thank Professor McMichael for suggesting this study and his continued help and advice, and also the Physicians of Hammersmith Hospital for their assistance and co-operation.

\section{REFERENCES}

Adams, R. (1827). Dublin Hosp. Rep., 4, 436.

Benson, C. (1836). Dublin J. med. chem. Soc., 8, 324.

Bloomfield, R. A., Lauson, H. D., Cournand, A., Breed, E. S., Richards, D. W. (1946). J. clin. Invest., $25,639$.

Carvallo, R. (1946). Arch. Inst. Card. Mexico, 16, 531.

Davies, C. (1827). Dublin Hosp. Rep., 4, 272.

Gerhardt, D. (1902). Arch. exp. Path. Pharmak., 47, 250.

Graves, - (1833). Lond. med. surg. J., 3, 74.

Groedel, F. M. (1946). The Venous Pulse and its Graphic Recording. New York.

King, T. W. (1837). Guy's Hosp. Rep., 2, 104.

Lagerlöf, H., and Werkö, L. (1948). Cardiologia, 13, 17.

Laubry, Ch., and Routier, D. (1928). Bull. Med., 3, 91.

Mackenzie, J. (1908). Diseases of the Heart. Oxford University Press, London.

McCord, M. C., and Blount, S. G. (1952). Amer. Heart J., 44, 671.

Messer, A. L., Hurst, J. W., Rappaport, M. V., and Sprague, H. B. (1950). Circulation, $1,388$.

Rihl, J. (1907). Berl. Klin. Wschr., 44, 825. 
Rosenbach, O. (1878). Arch. exp. Path. Pharmak., 9, 1.

Sherlock, S. (1951). Brit. Heart J., 3, 273.

Soulié, P., Bouvrain, Y., and Di Matteo, J. (1952). Bull. Soc. Med., 4, 332.

Teufl, R. (1936). Wien. med. Wschr., 11, 288.

Ward, T. O. (1832). Lond. med. Gaz., 10, 376.

Wiggers, C. J. (1923). Circulation in Health and Disease. Philadelphia. 